\title{
RANDOMIZED CONTROL TRIAL FOR RADIOGRAPHIC ASSESSMENT OF TWO DIFFERENT ATTACHMENT MATERIAL RETAINING BAR SUPPORTED OVERDENTURE
}

\author{
Rami M. Ghali1* and Hesham E. Alameldeen**
}

\begin{abstract}
Objectives: this research was carried out to evaluate two different attachment material retainers bar supported overdenture regarding the peri-implant crestal bone level changes.

Materials and methods: Twenty edentulous female patients participated in this study For each patient 3 endosteal implants were inserted in the interforamenal region using computer guided surgical Stent. Four month later implants were uncovered, healing abutments were secured and then transfer coping with long heads were used to make open tray impression technique. Plastic abutments were secured to implant analogue then plastic bars was attached to plastic abutments using durallay with two semi precision attachments at the distal ends of the bar. Then the bar was casted. Patients were divided into two groups. Group one the CAD CAM wax pattern of housing and the female part of the attachments were casted using nickel chrome alloy. Group two patients the CAD CAM wax pattern of housing and the female part of the attachments were transferred into Acetal resin using the injection molded technique. Then per implant crestal bone level were assessed using long cone parallel technique after six, twelve and twenty four months and then statistically analyzed.
\end{abstract}

Results: This study showed that there was a statistically insignificant difference in between follow up periods for each group and between groups in between follow up period in interval 12-24 and significant difference between two groups after 24 months.

Conclusion: Based on the results obtained from this study it can be concluded that acetal resin is favorable long term solution for implant retained mandibular overdentures

\section{INTRODUCTION}

Oral rehabilitation with an overdenture on splinted or unsplinted implants is considered the standard care in cases of mandibular edentulism. Numerous studies have shown that the mandibular implant over denture is a simple and effective solution and leads to significant improvement of patient-based outcomes as compared to conventional dentures. ${ }^{[1-3]}$

\footnotetext{
* Associate Professor of Prosthodontics,Faculty of Dentistry Ain Shams University.

** Lecturer of Prosthodontics, Faculty of Dentistry,Future University.
} 
The use of a wide variety of attachment systems, including stud, magnet and bar attachments have proven both clinically predictable and effective results. The design of attachments should provide equal implant-tissue support and optimum force distribution around the implants to allow bone loading within physiologic levels. ${ }^{[4,5]}$

Implants splinted together with bars may decrease the risk of overload to each implant as a result of a greater surface area, load sharing between implants and improved biomechanical distribution. [6-8] The bar ability to minimize the potential for micromotion at the bone-implant interface may help successful osseointegration of immediately loaded implants dentures. ${ }^{[9,10]}$ Bar attachment are classified according to their biomechanical behavior into rigid and resilient attachment. In comparison to resilient bar attachment, rigid anchoring of removable prostheses creates stable occlusal plane, reduces loading of denture-bearing areas, and minimizes posterior mandibular ridge resorption. One of the major drawbacks of rigid bar attachment is over loading of the abutments, however resilient bar attachment encourages torsion-free load transmission to implants dentures. The main disadvantages of bar attachments are the need for a large prosthetic space and the risk of mucositis due to an inadequate oral hygiene under the $\operatorname{bar}^{[11]}$.

Retention can be obtained directly from the bar by means of a clip, resilient liners or retention can be obtained from stud attachments attached the body of the bar. The use of bar with stud attachment is favorable when there is insufficient inter-implant distance to accommodate for a clip or when movement is required with a bar having two or more non-linear segments. ${ }^{[12]}$

In these situations the bar serves the purpose of implant splinting and prosthesis support while retention is obtained from two or more stud attachments placed on top of the bar, at its distal end, on the labial or lingual aspect or at a combination of these locations. The location of the stud attachment depends mainly on the available inter-arch distance and the implant tilt. ${ }^{[12-14]}$ In limited inter arch spaces the stud attachment cannot be placed on top of the bar. Instead ERA (extra-coronal resilient attachment) can be placed on its distal end. ${ }^{[12]}$

Bar attachment employs a rigid interconnection between the implants using a cast metal bar attachment. The overdenture is fabricated to passively fit and attach to the bar. The connection between the bar and denture base provides the attachment's retentive quality. This method is suitable for two or more implants, but is the method of choice when more than two implants are used. ${ }^{[15]}$

Comparing three different types of attachments (magnet, ball and straight bar with clip) used for connection between implants in implant supported overdenture showed that the state of connection did not influence the clinical success of implants. Separate attachments provide lower retention capacity than bars to retain overdenture, but the assessment of patient does reflect this difference. ${ }^{[16]}$

A variety of prefabricated bar designs exist. However, due to a lack of accurate adaptation of the denture base to the bar superstructure, rotation and lateral movement of the denture are unavoidable. Electrical Discharge Machining (EDM) and spark erosion can be used to improve the fit of overdenture framework, but this procedure is costly and technique-sensitive. ${ }^{[17,18]}$ Milled bars have been suggested as a less expensive alternative to EDM. Implant-supported milled bars are bars with precision attachments and rigid anchorage, made by casting, electro erosion or CAD-CAM (computeraided design and computer- aided manufacturing). Traditional castings have a major limitation inherent in the process, which is distortion of the casting with increasing size of the pattern.CAD/CAM fabrication of bars and frameworks has resulted in elimination of distortion, better fit, and fewer fabrication steps. ${ }^{[17,18]}$ 
Unlike prefabricated bars, a custom-fabricated bar could be accurately milled to develop guide planes that allow accurate adaptation of the denture base to the milled bar providing stability and resistance against rotational and lateral forces. Custom made bars are also more likely to follow the ridge shape when pronounced ridge curvature is encountered, without invading the tongue space. ${ }^{[18-20]}$

In a randomized, controlled clinical trial, 110 edentulous patients with atrophic mandibles were treated using three different treatment strategies: a mandibular overdenture supported by two implants with ball attachments, two implants with an interconnecting bar, or four interconnected implants. The results showed that no significant difference was found between the three treatment strategies, it was concluded that simple implant treatment such as an overdenture retained by two ball attachments is sufficient. ${ }^{[21]}$ However there is some evidence that stresses and loads are distributed more uniformly if the implants are connected by a bar. ${ }^{[7]}$

Thermoplastic resins have been used in dentistry for over 50 years. Since that time their applications have continued to grow, and the interest in nylon based materials have increased ${ }^{[22-24]}$ The "TSM ACETAL DENTAL" is a thermoplastic techno polymer with a monomer-free crystalline structure and is a product of formaldehyde polymerization. Its characteristics are: high resistance to abrasion, excellent tensile and shock strength, high elastic memory, low thermal conductance and rigidity. Furthermore, it is neither allergenic, nor toxic and resists occlusal wear. ${ }^{[25]}$

These features, coupled with remarkable aesthetic performance, make "TSM Acetal Dental" a good substitute for acrylic resins and metals in many prosthetic applications. These characteristics make it an ideal material for pre-formed clasps for partial dentures, single pressed unilateral partial dentures, partial denture frameworks, provisional bridges, occlusal splints, and implant abutments..
It was further modified by reinforcement with glass fiber and glass spheres to increase its potential use as a denture base material wear. ${ }^{[25-27]}$

Acetal resins are highly versatile engineering polymers that bridge the gap between metals and ordinary plastics. Because they offer the strength of metal and the flexibility and comfort of plastic, they make an ideal material for the fabrication of dental prostheses. However the effect of this material as an implant supported base material regarding the functional loads onto the implants supporting tissues has not been sufficiently assessed.

Objective evaluation of the treatment outcome is paramount in order to ensure predictability and excellent long-term prognosis. Hence, the aim of the present study is to evaluate and compare periimplant crestal bone level changes around implants in edentulous patients rehabilitated with implantsupported bar overdenture and retained by different attachments materials attached to the body of the bar distally constructed by CAD CAM technology.

\section{MATERIALS AND METHODS}

Twenty edentulous male patients with a mean age of 65 year complaining from insufficient retention of their mandibular denture were selected to participate in this study. Exclusion criteria were: $\mathrm{V}$ - shaped edentulous ridge, insufficient bone volume in the inter-foraminal region of the mandible with a minimum length of $12 \mathrm{~mm}$ and $7 \mathrm{~mm}$ width , mucosal lesions, class II and III ridge relationship , insufficient interarch space and patients suffering from neuromuscular disorders,. temporomandibular joint disorders, Un-controlled diabetes, osteoporosis, parafunctional habits, smokers and administrative or physical considerations that would seriously affect the surgical procedure were also excluded.

A preoperative radiographic examination was made using Cone beam computed tomography (CBCT) to trace the exact position of the mandibular neurovascular bundle and the available bone height, width and quality. 
All the patients were rehabilitated with an implant-supported bar overdenture. For All patients three implants $(3.7 \mathrm{~mm})$ in diameter $(11 \mathrm{~mm})$ in length were inserted in the inter-foraminal region using computer guided surgical template.

Four months later, the implants were uncovered, healing abutments were screwed for two weeks, then transfer copings with long head screws were used following open tray impression technique using medium body elastomer ( $3 \mathrm{M}$ impregum) in a special tray, Implants analogue were then screwed to the transfer copings, impression was poured using hard dental stone. (Fig 1). Plastic abutment were secured to the implant analogues then plastic bars (10mm in height and square in cross section) was attached to the plastic abutment using durallay (with two semi precession attachment at the distal ends of the bar(OT vertical rehin).

The whole assembly was tried in the patient mouth and checked for passivity by one screw tab technique, then cast, and secured over the implant analogues on the master cast. Cast bar was tried in the patient mouth to assure passive fit (Fig2) and $\mathrm{x}$-ray was taken to assure intimate contact between the assembly and the implants (Fig3)

The cast bar was scanned using bench laser scanner(smart optic) to obtain virtual model with the bar, the housing on the top of the bar and the female

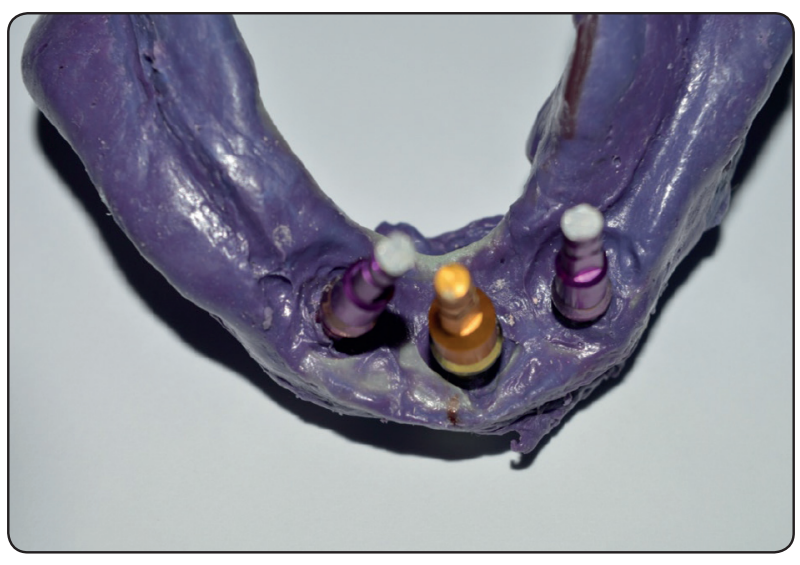

Fig. (1) Impression with transfer copings and implant analogues in place

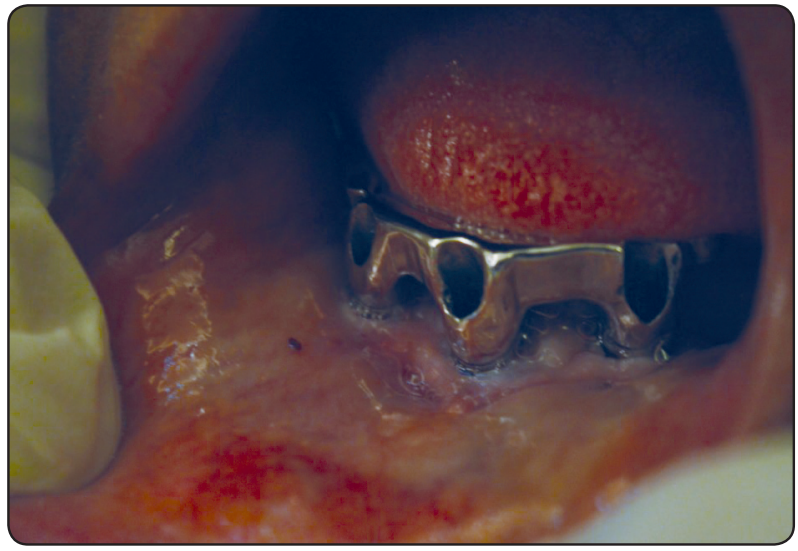

Fig. (2) Showing bar assembly in patient mouth

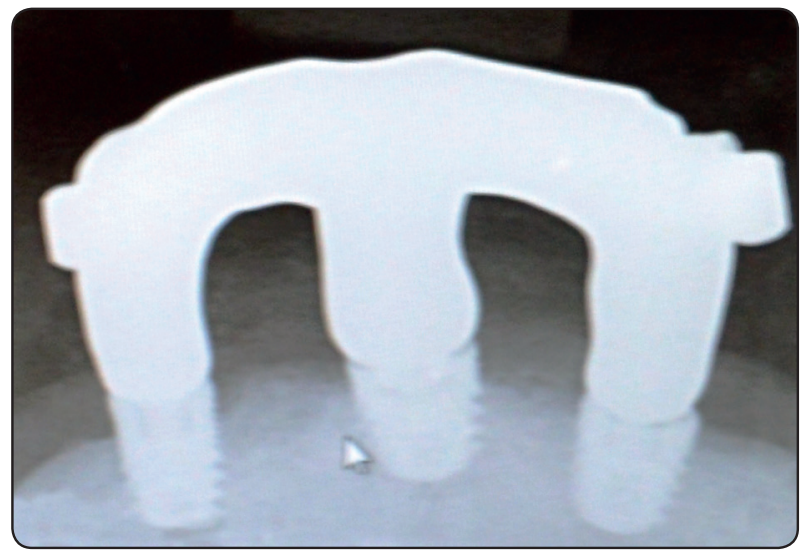

Fig. (3) Showing x-ray to assure intimate contact between bar and implant.

part of the attachment was designed using computer aided designing (CAD) (exocad) then milled into wax (shera) by computer aided manufacturing (CAM) (DWX-50Roland).

Patients were randomly divided into two groups

Group one patients: The CAD CAM wax pattern of housing and the female part of the attachments were casted using nickel chrome alloy with space around the distal attachment to accommodate the plastic sleeve (Fig4).

Group two patients: The CAD CAM wax pattern of housing and the female part of the attachments were transferred into acetal resin using the injection molded technique (Fig 5\&6) 


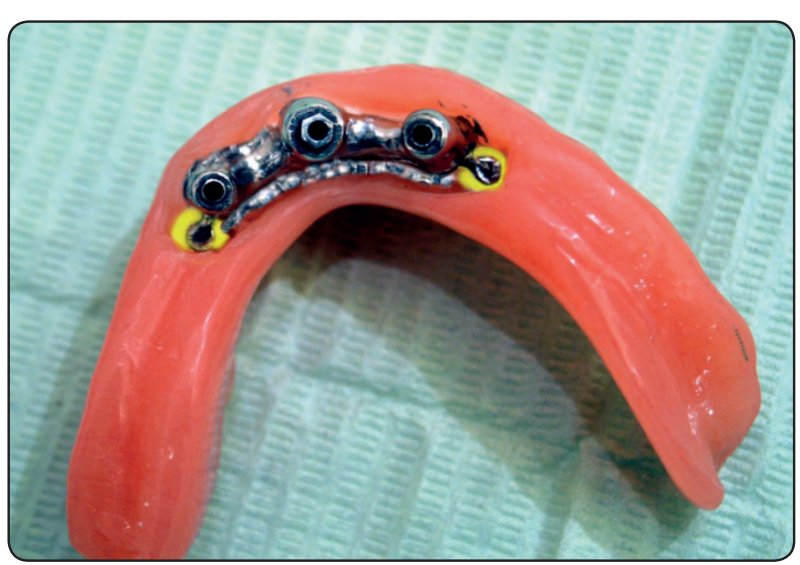

Fig. (4) Showing complete overdenture with female metal housing and elastic attachment and the bar with the distal male part of the attachment.

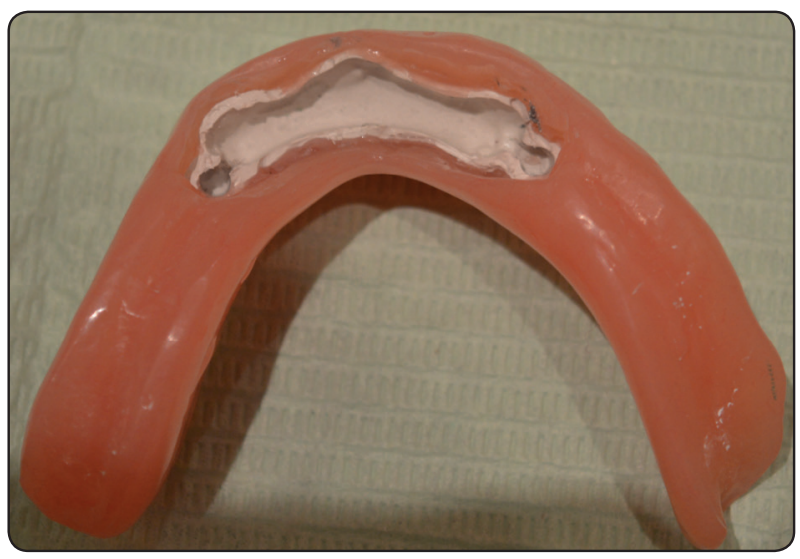

Fig. (5) Showing complete overdenture with female Acetal resin attachment.



Fig. (6) Showing complete overdenture with female Acetal resin attachment and the bar with the distal male part of the attachment.
Jaw relation was registered and over dentures were constructed to all the patients following the same basic principles. Centric occlusion was developed at centric relation. Modified cusped acrylic teeth were used and balanced on semi-adjustable articulator for centric and eccentric positions following the medial positioned lingualized concept of occlusion. Prior to insertion, dentures were clinically remounted to refine the occlusion; to ensure free anterior contact in centric and free non-interfering contact during all excursive mandibular movements.

Patients were recalled frequently for postinsertion inspection and adjustment. Follow up visits were scheduled at time of denture insertion, six, twelve and twenty four month after overdenture insertion for inspection of the prosthesis and collection of the data (radiographic evaluation). Clinical evaluation for the cases was also carried out to inspect implants.

Radiographic evaluation: Peri-implant crestal bone level changes were assessed using intraoral radiographs taken with the standardized long cone paralleling technique using custom made acrylic template and the Rinn-XCP system. ${ }^{[28]}$ After performing the needed post insertion adjustment, periapical radiographs were taken after six months, twelve months and twenty four month to complete a period of two years follow up period. A piece of wire was embedded in the acrylic template was used as a reference point to assess marginal bone height changes at the mesial and distal aspect of each implant using Digora software system. Each time patients were recalled, dentures were evaluated and occlusal adjustment was performed.

\section{RESULTS}

All the data was collected and tabulated. Statistical analysis was performed by Microsoft Office 2010 (Excel) and Statistical Package for Social Science (SPSS) version 20. 
The significant level was set at $\mathrm{P} \leq 0.05$. Kolmogorov-Smirnova and Shapiro-Wilk tests was used to assess data normality and data was assumed normally distributed. ANOVA for repeated measures test was used to compare between follow up periods within groups and when statistically significant it was followed by simple main effect analysis with Bonferioni correction. One way ANOVA test was used to compare between groups at different follow up periods.

Table (1) represents the mean value of periimplant bone loss and their level of significance during the follow up period for group I patients and group II patients between consecutive intervals and from loading to each recall appointment. The data revealed that the mean value of peri-implant bone loss for group I patients between consecutive interval loading - 6 months (0.34), interval 6- 12 months (0.25) and interval $12-24$ months (0.15). .A total change of (0.59) and (0.74) was calculated at the end of the twelve months and twenty four months follow up period respectively. Although the data obtained from table 1 revealed increase in the amount of peri-implant bone loss during the follow up period, statistical analysis of the data following ANOVA test for repeated measures revealed in significance difference in between follow up periods.

The data revealed that the mean value of periimplant bone loss for group II patients between consecutive interval loading - 6 months (0.31), interval $6-12$ months (0.17) and interval $12-24$ months (0.11). . A total change of (0.48) and (0.59) was calculated at the end of the twelve months and twenty four months follow up period respectively. Although the data obtained from table 1 revealed increase in the amount of peri-implant bone loss during the follow up period, statistical analysis of the data following ANOVA test for repeated measures revealed in significance difference $(\mathrm{P} \geq$ 0.05 ) between groups in between follow up periods.
The difference in the means of change in periimplant bone loss between the two studied groups showed a higher change in bone for implants bearing between consecutive intervals, loading - 6 months 6 - 12 months and interval $12-24$ months One way ANOVA test revealed insignificance difference $(\mathrm{P}<$ 0.05 ) between groups. At the end of the 12 months and 24 months follow up periods, the mean value of peri-implant bone loss for group I patients was 0.59 and 0.74 while for group II patients was 0.48 and 0.59 One way ANOVA test revealed significance difference $(\mathrm{P}>0.05)$ between groups

TABLE (1) Mean and standard deviation values of peri-implant bone loss of the studied groups at different follow up periods.

\begin{tabular}{|c|c|c|}
\hline $\begin{array}{c}\text { Group } \\
\text { Interval }\end{array}$ & $\begin{array}{c}\text { Group I } \\
\text { Mean, SD d1,d2 }\end{array}$ & $\begin{array}{c}\text { Group II } \\
\text { Mean, SD d1 ,d2 }\end{array}$ \\
\hline Loading------6 m & $0.34 \pm 0.08^{\mathrm{a}--\mathrm{a}}$ & $0.31 \pm 0.04^{\mathrm{a}--\mathrm{a}}$ \\
\hline 6mon------12 m & $0.25 \pm 0.07^{\mathrm{a}-\mathrm{a}}$ & $0.17 \pm 0.021^{\mathrm{a}-\mathrm{-a}}$ \\
\hline Loading------12 m & $0.59 \pm 0.21^{\mathrm{a}-\mathrm{a}}$ & $0.48 \pm 0.19^{\mathrm{a}--\mathrm{ab}}$ \\
\hline $12 \mathrm{mon}------24 \mathrm{~m}$ & $0.15 \pm 0.04^{\mathrm{a}---\mathrm{a}}$ & $0.11 \pm 0.05^{\mathrm{a--a}}$ \\
\hline Loading------24 m & $0.74 \pm 0.18^{\mathrm{a}--\mathrm{a}}$ & $0.59 \pm 0.21^{\mathrm{a}--\mathrm{ab}}$ \\
\hline
\end{tabular}

AONVA for repeated measures, similar superscript letters indicate non significant difference.

d1 within group d2 between groups

\section{DISCUSSION:}

Implant supported over dentures are today regarded as a routine option for the totally edentulous mandible. This trend in the prosthodontic literature has led to a significant shift in therapeutic philosophy regarding restoration of the edentulous patient.

In accordance with other comparative studies, this study did not demonstrate any significant difference in mean marginal loss after 2 years using 
a supporting bar with a ball retentive system or acetal. ${ }^{[29,30]}$

In both groups, only very small changes in marginal bone level were observed from 1 to the 2 -year registration. Evident peri-implant bone level change was, however, observed during the first year of loading. This bone loss may be explained by the surgical trauma when placing the implants and by the bone remodeling after placement.

The use of bar with accurately developed guide planes allowed intimate adaptation of the denture base to the bar providing a great amount of stability and resistance against rotational and lateral forces. Incorporating a distal attachment provided the patient with sufficient retention to prevent vertical movement of the denture. ${ }^{[19,20]}$

Critical to the long term success of implant based restorations is careful consideration of the passive fit. Following osseointegration and placement of a final restoration, both functional and parafunctional loads can be transferred to the implants that may affect remodeling of the bone-implant interface. In the case of splinted implant restorations, it is possible that the restoration itself may transmit strain to the bone-implant interface. Ideally, the restoration would fit passively on the supporting implants, thus minimizing strain and the concomitant biological response.

The concept of clinically acceptable fit using $\mathrm{CAD} / \mathrm{CAM}$ frameworks incorporates the goal of minimizing both biologic complications and mechanical complications. Clinically acceptable fit in combination with control over the occlusal forces is important for ensuring success of the implants and the restoration can account for the results of this study ${ }^{[31]}$

The strict oral hygiene program that the patients in the present study followed influenced the periimplant outcome positively in both groups this may explain the healthy periimplant conditions reported after
2 years.Another factor contributing to the good results may be the splinting of the implants with the bar. By splinting, the implants work as a group rather than as single units, thereby compensating for lateral forces ,one factor contributing to the good results is probably the effort given to achieving high primary implant stability by using thinner drills and/or tapered implant designs. Primary implant stability is a function of local bone quality and quantity, implant design, surgical technique used, and precise fit in the bone; and that is a very important parameter in achieving osteointegration and long term success in dental implants ${ }^{[32]}$. A major factor affecting the primary stabilities of implants is bone density, according to Misch $^{(13,34)}$. The capacity of bone to withstand physiologic loads differs according to the bone quality, with the highest strength for D1 bone and the lowest strength with D4 bone quality. In addition, it was observed that the length and diameter of implants have an influence on the primary stability, placement, and removal torque values. Self-tapping also influences their stability inside the bone and plays a significant role in osteointegration ${ }^{[3,33]}$.

The results of this study supports the opinion that bone density is one of the major determining factors for the high success rate since all the selected patients exhibited D1 bone density. A logical explanation lies in the functional surface area of contact between the implant and the bone, in addition to the high mechanical tolerance for D1 bone quality to functional load. Placement of implants in the anterior region of the mandible plays a major role in the overall success, because this area has the highest bone quality.

There are several reports that Acetal resin have a sufficiently high resilience and modulus of elasticity that controls the excessive torqueing forces that may act on the implants and can explain the favorable outcome of peri-implant loss changes detected in this study ${ }^{[22-24]}$. 


\section{CONCLUSION}

Based on the results obtained from this study it can be concluded that acetal resin is favorable long term solution for implant retained mandibular overdentures

\section{REFERENCES}

1. Banton, B. and M. Henry, Overdenture retention and stabilization with ball-and-socket attachments: principles and technique. Journal of dental technology: the peer-reviewed publication of the National Association of Dental Laboratories, 1997. 14(7): p. 14-20.

2. Naert, I., G. Alsaadi, and M. Quirynen, Prosthetic aspects and patient satisfaction with two-implant-retained mandibular overdentures: a 10-year randomized clinical study. International Journal of Prosthodontics, 2004. 17(4).

3. Tokuhisa, M., Y. Matsushita, and K. Koyano, ln Vitro Study of a Mandibular Implant Overdenture Retained with Ball, Magnet, or Bar Attachments: Comparison of Load Transfer and Denture Stability. International Journal of Prosthodontics, 2003. 16(2).

4. Trakas, T., et al., Attachment systems for implant retained overdentures: a literature review. Implant dentistry, 2006. 15(1): p. 24-34.

5. Van Kampen, F., et al., Retention and postinsertion maintenance of bar-clip, ball and magnet attachments in mandibular implant overdenture treatment: an in vivo comparison after 3 months of function. Clinical oral implants research, 2003. 14(6): p. 720-726.

6. Akca, K., et al., Bone strains around immediately loaded implants supporting mandibular overdentures in human cadavers. International Journal of Oral \& Maxillofacial Implants, 2007. 22(1).

7. Mericske-stern, R., M. Piotti, and G. Sirtes, 3-D in vivo force measurements on mandibular implants supporting overdentures. A comparative study. Clinical oral implants research, 1996. 7(4): p. 387-396.

8. Misch, C.E., et al., Rationale for the application of immediate load in implant dentistry: part II. Implant Dentistry, 2004. 13(4): p. 310-321.

9. Lorenzoni, M., et al., In-patient comparison of immediately loaded and non-loaded implants within 6 months. Clinical oral implants research, 2003. 14(3): p. 273-279.
10. Alfadda, S.A., N.J. Attard, and L.A. David, Five-year clinical results of immediately loaded dental implants using mandibular overdentures. International Journal of Prosthodontics, 2009. 22(4)

11. Preiskel, H.W., Overdentures made easy: a guide to implant and root supported prostheses. 1996: Quintessence Publishing Company.

12. Steffen, R.P., V. White, and N. Robert Markowitz, The use of ball-clip attachments with an implant-supported primary-secondary bar overdenture. Journal of Oral Implantology, 2004. 30(4): p. 234-239.

13. Misch, C., Dental implant prosthetics, Mosby, St. Louis Missouri, 2005: p. 32-41.

14. Babbush, C.A., Dental implants: principles and practice. 1991: WB Saunders Company.

15. Burns, D.R., Mandibular implant overdenture treatment: consensus and controversy. Journal of Prosthodontics, 2000. 9(1): p. 37-46.

16. Naert, I., et al., A 5-year prospective randomized clinical trial on the influence of splinted and unsplinted oral implants retaining a mandibular overdenture: prosthetic aspects and patient satisfaction. Journal of oral rehabilitation, 1999. 26(3): p. 195-202.

17. Ercoli, C., et al., Alternative procedure for making a metal suprastructure in a milled bar implant-supported overdenture. The Journal of prosthetic dentistry, 1998. 80(2): p. 253-258

18. Brudvik, J.S. and K. Chigurupati, The milled implant bar: an alternative to spark erosion. Journal-Canadian Dental Association, 2002.68(8): p. 485-488

19. Galindo, D.F., The implant-supported milled-bar mandibular overdenture. Journal of Prosthodontics, 2001 . 10(1): p. 46-51.

20. Al-Fadda, S.A., G.A. Zarb, and Y. Finer, A comparison of the accuracy of fit of 2 methods for fabricating implantprosthodontic frameworks. International Journal of Prosthodontics, 2007. 20(2).

21. Van Kampen, F., et al., The influence of various attachment types in mandibular implant-retained overdentures on maximum bite force and EMG. Journal of Dental Research, 2002. 81(3): p. 170-173.

22. Kohli, S. and S. Bhatia, Polyamides in dentistry. Int J of Scientific Study, 2013. 1(1): p. 20-5. 
23. Takabayashi, Y., Characteristics of denture thermoplastic resins for non-metal clasp dentures. Dental materials journal, 2010. 29(4): p. 353-361.

24. Thakral, G., et al., Flexible Partial Dentures-A hope for the Challenged Mouth. People's Journal of Scientific Research, 2012. 5(2): p. 17-21.

25. Phoenix, R.D., et al., Evaluation of mechanical and thermal properties of commonly used denture base resins. Journal of Prosthodontics, 2004. 13(1): p. 17-27.

26. John, J., S.A. Gangadhar, and I. Shah, Flexural strength of heat-polymerized polymethyl methacrylate denture resin reinforced with glass, aramid, or nylon fibers. The Journal of prosthetic dentistry, 2001. 86(4): p. 424-427.

27. Ozkan, Y., et al., A study to assess the colour stability of acetal resins subjected to thermocycling. The European journal of prosthodontics and restorative dentistry, 2005. 13(1): p. 10-14.

28. Frederiksen, N.L., Diagnostic imaging in dental implantology. Oral Surgery, Oral Medicine, Oral Pathology, Oral Radiology, and Endodontology, 1995. 80(5): p. 540-554.
29. Batenburg, R.H., et al., Mandibular overdentures supported by two or four endosteal implants: a prospective, comparative study. International journal of oral and maxillofacial surgery, 1998. 27(6): p. 435-439.

30. Naert, I., et al., A 5-year randomized clinical trial on the influence of splinted and unsplinted oral implants in the mandibular overdenture therapy. Part I: peri-implant outcome. Clinical Oral Implants Research, 1998. 9(3): p. 170177 .

31. Takahashi, T. and J. Gunne, Fit of implant frameworks: an in vitro comparison between two fabrication techniques. The Journal of prosthetic dentistry, 2003. 89(3): p. 256260 .

32. Mericske-stern, R., et al., Peri-implant mucosal aspects of ITI implants supporting overdentures. A five-year longitudinal study. Clinical Oral Implants Research, 1994. 5(1): p. 9-18.

33. Khoury, F. and A. Happe, Interim implants in extensive bone transplantation procedure: results of a clinical study. Implantologie, 2000. 9: p. 375-387. 